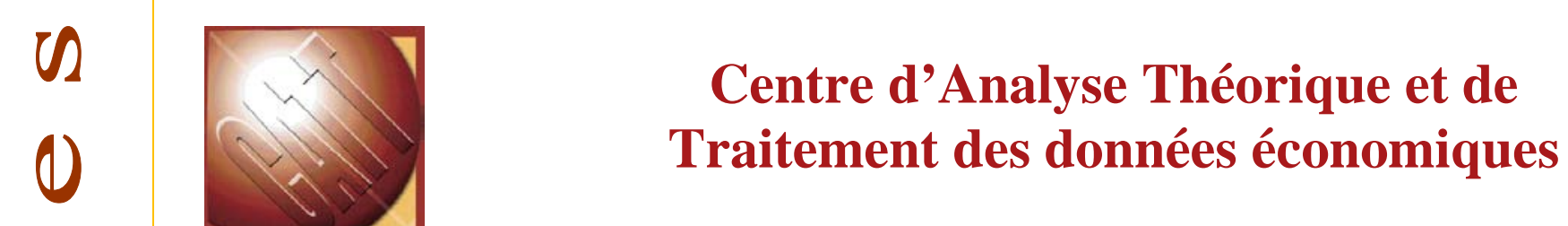

0

CATT WP No. 4 January 2012

IMPACT DES TRANSFERTS DE FONDS SUR LE TAUX DE CHANGE REEL EFFECTIF EN TUNISIE

Khaled Chnaina

Farid Makhlouf 


\title{
Impact des Transferts de Fonds sur le Taux de Change Réel Effectif en Tunisie
}

\author{
Chnaina Khaled \\ Centre d'Analyse et Traitement Théorique des Données Economiques \\ Domaine universitaire - Avenue du Doyen Poplawski - BP1633 - 64016 PAU cedex \\ khaled.chnaina@etud.univ-pau.fr \\ Makhlouf Farid \\ Centre d'Analyse et Traitement Théorique des Données Economiques \\ Domaine universitaire - Avenue du Doyen Poplawski - BP1633 - 64016 PAU cedex \\ farid.makhlouf@univ-pau.fr
}

Résumé

Les transferts de fonds vers la Tunisie constituent une source de devises non négligeable, Ils ont augmenté d'une manière rapide notamment la dernière décennie. Ces devises engendrées par les migrants tunisiens, peuvent causer le phénomène de syndrome hollandais. Le but de ce travail est d'étudier l'impact des ces transferts sur taux de change réel effectif. En estimant des relations de cointégration avec ruptures structurelles par l'intermédiaire de la méthode de Johansen et al. (2000) et Saikkonen et Lütkepohl (2000) et Saikkonen et al. (2004), nous avons trouvé qu'une augmentation de $1 \%$ de ratio des transferts de fonds sur PIB provoque une appréciation de taux de change réel d'équilibre de $0,39 \%$. Ce qui confirme l'hypothèse de syndrome hollandais dans l'économie tunisienne.

Mots clés: Transferts de Fonds, Taux de Change Réel Effectif, Ruptures Structurelles, Cointégration, Tunisie.

\begin{abstract}
Remittances in Tunisia constitute a main foreign flow, they have increased rapidly particularly over the past decade. This devises which generated by Tunisian migrants can induce Dutch disease. The aim of this study is to analyze the relationship between remittances and reel effective exchange rate. By estimating cointegration relations with structural breaks through the method of Johansen et al. (2000) and Saikkonen-Lütkepohl (2000) Saikkonen et al. (2004), we found that an increase in worker's remittances of $1 \%$ point of GDP is associated with an appreciation of Tunisia's real effective exchange rate by $0.39 \%$. This result approves the hypothesis of Dutch disease in Tunisia.
\end{abstract}

Keywords: Remittances, Reel Effective Exchange Rate, Structural Breaks, Cointegration, Tunisia.

\footnotetext{
${ }^{1}$ Les auteurs tiennent à remercier le Professeur Serge Rey, pour ses commentaires sur les versions préliminaires de ce papier. Toutes les erreurs qui subsistent sont imputables aux auteurs.
} 


\section{1) Introduction}

Le débat concernant l'impact des transferts de fonds des migrants sur les économies des pays d'origine est en pleine effervescence. Cette ressource financière engendrée par la migration internationale peut servir à alléger certaines contraintes budgétaires des ménages dans les pays d'origine. Elle peut les aider à améliorer leurs niveaux de vie. Par ailleurs, elle peut aussi engendrer certains effets non souhaités sur les économies bénéficiaires. En effet, la littérature économique récente s'intéresse aux effets néfastes des transferts de fonds sur le plan macro et micro-économique. En outre ces effets négatifs peuvent se manifester sous forme d'un phénomène connu sous le non de syndrome hollandais Fajnzylber et López, (2007). De même, cet effet est particulièrement important dans les petits pays Kapur, (2004). Ce phénomène est analysé par (Corden et Neary, 1982; Corden, 1984) dans le cas d'une rentrée massive des devises. Ils peuvent apporter des nouveaux ajustements sur l'économie tunisienne, en particulier par l'effet dépense ${ }^{2}$. Cependant, Grabel (2008) souligne que les effets macro-économiques des transferts sont complexes.

Dans ce papier, nous allons contribuer et enrichir ce débat en étudiant le cas de la Tunisie. L'objectif de notre travail est de vérifier si les transferts de fonds provoquent une appréciation de Taux de Change Réel Effectif (TCRE), soit une perte de compétitivité de secteur échangeable non abrité. La croissance accrue des transferts de fonds, notamment depuis 2000 (annexe figure1), nous motive à diagnostiquer la présence d'un tel phénomène. A cet égard le taux de croissance moyen annuel est 8,05\% pour la période (2000-2009) ${ }^{3}$. Ils avoisinent les 2 milliards de dollars en 2009. Ils sont engendrés en grosso modo par des tunisiens résidents en Europe. Selon les statistique de la Banque Mondiale (2011), il y a environ 6.3\% de population totale tunisienne vivent à l'étranger en 2009. Parmi les principales destinations des migrants tunisiens nous citons, la France, l'Italie, l'Allemagne et la Libye pays frontalier.

Les transferts de fonds sont considérés comme étant une source de devises moins volatiles, notamment par apport aux autres flux financiers et peuvent augmenter dans les périodes de crises (Ratha ,2003; Mughal et Makhlouf, 2011). Dans le cas de la Tunisie la part des transferts de fonds sur le PIB reste stable dans le temps par rapport aux IDE, (annexe,

\footnotetext{
${ }^{2}$ La figure 3 dans l'Annexe, montre une forte corrélation entre les transferts de fonds et la consommation finale des ménages tunisiens.

${ }^{3}$ A partir des données de la Banque Mondiale (WDI) 2011.
} 
figure2). Ainsi, elle est supérieure à celle des IDE et les Aides sauf pour l'année 2006 où la part des IDE sur le PIB a touché la barre des $10 \%$ (annexe, figure2).

La suite de ce travail sera organisée comme suit : après avoir présenté la littérature associé à la problématique dans la section 2 et présenter le modèle adapté au cas de la Tunisie dans la section 3, nous procéderons à l'analyse empirique dont nous allons expliquer la stratégie et le choix d'estimateur, ainsi que discuter les résultats. Enfin, dans la section cinq, nous conclurons ce travail.

\section{2) Revue de la littérature}

La théorie de syndrome hollandais est associée à une appréciation de taux de change suite à une entrée massive des capitaux étrangers. Cette théorie formulée au départ pour les pays développés, notamment à partir de la découverte de gaz en Holland dans les années 50. La pertinence de cette théorie a poussé les économistes à l'appliquer pour les pays en développement. Cependant, l'application de cette théorie pour les pays en développement peut donner des résultats différents par rapport aux pays développés. Cette différence est due pour une partie aux différences de régime de change. En effet, Krugman et Obstfled (2006, P 664) soulignent certaines rigidités des régimes de change des pays en développement. Dans le cas des transferts de fonds, Vargas-Silva(2007) note que leurs effets sur le TCRE restent encore ambigus. In hoc senso, Gabrel (2008) considère que les effets à court terme des transferts de fonds sont similaires par rapport à d'autres flux financiers. Par ailleurs, leurs impacts à long terme sont différents selon les politiques économiques engagées dans les pays bénéficiaires. Alors qu'une rentrée massive de devises étrangères pourrait provoquer l'appréciation de taux de change et la baisse de la compétitivité. Bourdet et Falck (2006) ont analysé les effets de transferts sur le Cap-Vert, et ont montré que les aides et les transferts de fonds ont un effet négatif sur la compétitivité. Lartey et al. (2008), en utilisant des données de panel ont trouvé que les transferts de fonds engendrent un effet de dépense et celui de réallocation de ressources. Amuedo-Dorantes et Pozo (2004) ont trouvé qu'une augmentation de $100 \%$ des envois de fonds va engendrer une augmentation de $22 \%$ de taux de change réel, en utilisant les données de panel pour 13 pays d'Amérique Latine et Caraïbe. Makhlouf et Mughal (2010), en utilisant la statistique bayésienne, ont trouvé que les transferts de fonds provoquent le phénomène de syndrome hollandais en Pakistan. Singer (2008) est convaincu que les transferts de fonds des migrants exercent des pressions sur le choix de régime de taux de change. Barajas et al. (2010) en utilisant la technique de cointégration pour des données de 
panel ont trouvé une appréciation de TCRE suite à un choc des transferts de fonds. Contrairement, Mongardini and Rayner (2009) ont étudié le même phénomène pour des pays d'Afrique Subsahariens, leurs résultats indiquent que les transferts de fonds ne causent pas l'appréciation de TCRE. Aussi l'étude de Rajan et Subramanian (2005) ne trouve aucune preuve que les transferts de fonds des migrants affectent négativement la compétitivité.

\section{3) Modèle}

Le TCRE est considéré dans cette approche comme une trajectoire et non comme une valeur constante. Autrement dit, il est engendré par ses fondamentaux en situation d'équilibre interne et externe. En effet, notre modèle sera basé par les travaux de (Montiel, 1999; Hinkle et Montiel, 2001 ; Baffes et al., 1999 ; Edwards, 1989 ; Elbadawi et Soto, 1994) qui estiment le TCRE en fonction de ses fondamentaux. Cette approche est développée pour le cas d'un petit pays. Le TCRE noté (e) peut être défini comme un rapport entre le prix des biens non échangeables noté $\left(\mathrm{P}_{\mathrm{N}}\right)$ et le prix des biens échangeables noté $\left(\mathrm{P}_{\mathrm{E}}\right)$. Analytiquement nous avons : $e=\frac{P_{N}}{P_{E}}$

Une augmentation de ce taux, induite par la hausse des biens non échangeables indique une perte de compétitivité internationale qui est synonyme d'une appréciation réelle. En effet, le taux défini dans l'équation (1) correspond à la réalisation des deux équilibres simultanément : l'équilibre interne et l'équilibre externe. L'équilibre interne est atteint lorsque le marché des biens non échangeables s'est équilibré dans le présent et est supposé de s'équilibrer dans le futur. L'équilibre externe est atteint quand le solde du compte courant est compatible avec les flux des capitaux soutenables de long terme Williamson (1983).

Ce qui nous permet d'écrire l'équation suivante:

$$
Y_{n}(e, \rho)=C_{n}+G_{n}=(1-\theta) e C+G_{n}: \quad \frac{\partial Y_{n}}{\partial e} \succ 0, \frac{\partial Y_{n}}{\partial \rho} \prec 0 .
$$

Avec $Y_{n}$ est l'offre des biens non échangeables, $G_{n}$ et $C_{n}$ sont les dépenses des biens non échangeables publiques et privées respectivement, et $C$ représente les dépenses privées mesurées en termes de biens échangeables. Le paramètre $\theta$ représente la part de biens échangeables dans les dépenses privées totales et $\rho$ représente la productivité. La relation entre le TCRE et l'offre de biens non échangeables est négative. Une dépréciation du TCRE a 
pour effet d'augmenter l'offre de biens échangeables au détriment de l'offre de biens non échangeables grâce à l'amélioration de la compétitivité prix. Les mêmes effets sont associés à un choc de productivité : un déplacement de la main d'œuvre du secteur abrité vers le secteur exposé provoque une augmentation de l'offre de biens échangeables et une baisse de l'offre de biens non échangeables.

L'équilibre externe se réalise lorsque la position créditrice du pays $(\dot{f})$ atteint l'équilibre à l'état stationnaire. Il peut être formulé comme suit:

$\dot{f}=b+Z+r f=Y_{E}(e, \rho)-G_{E}-(\theta+\tau) C+Z+r f=0$

$f$ : La position extérieure nette et $r$ le taux de rendement des actifs étrangers, $z$ flux de capitaux à long terme supposés composés seulement des investissements directs étrangers (IDE). $b=$ balance commerciale $=$ production des biens échangeable - dépenses totales des biens échangeables donc, $b=Y_{E}(e, \rho)-\left(G_{E}+(\theta+\tau) C\right)$. $\tau$ représente les coûts de transaction et $G_{E}$ : dépenses publiques en biens échangeable. Nous avons aussi $\frac{\partial Y_{E}}{\partial e} \prec 0$ et $\frac{\partial Y_{E}}{\partial \rho} \succ 0$. Selon Montiel (1999), les dépenses privées sont négativement corrélés aux couts de transactions $(\tau)$ qui dépendent eux mêmes du taux d'intérêt nominal, c'est-à-dire du coût d'opportunité de détention de la monnaie.

Dans ce cadre d'analyse, nous allons étudier les effets des transferts de fonds des travailleurs tunisiens à l'étranger sur le TCRE d'équilibre en suivant Barajas et al. (2010). En effet, nous allons adopter l'hypothèse standard sur les transferts de fonds, i.e. ils sont exogènes. Sous cette hypothèse, les transferts de fonds interviennent que dans l'équilibre externe. En conséquence, ils apparaissent comme un terme additif dans la contrainte budgétaire donné par la condition d'équilibre extérieur. Suite à cette hypothèse, Barajas et al. (2010; pg: 9-10.) disent: "Accordingly, the effect of a permanent increase in the receipt of remittances is to shift the external balance locus to the right-an increase in remittance flows allows a higher level of household consumption to be consistent with external balance at an unchanged value of the real exchange rate. There are no direct effects on the internal balance locus, so the equilibrium is at $B^{\prime}$...." Ceci est le résultat standard où une augmentation du niveau des envois de fonds résulte une appréciation du TCRE d'équilibre et une augmentation de 
l'absorption privé. En conséquence seule la courbe d'équilibre extérieur $(\mathrm{EE})^{4}$ qui se déplace à droite vers B' (Figure ${ }^{\circ} 1$ ).

\section{Figure $n^{\circ}$ 1: effet d'une augmentation des envois de fonds sur le TCRE d'équilibre}

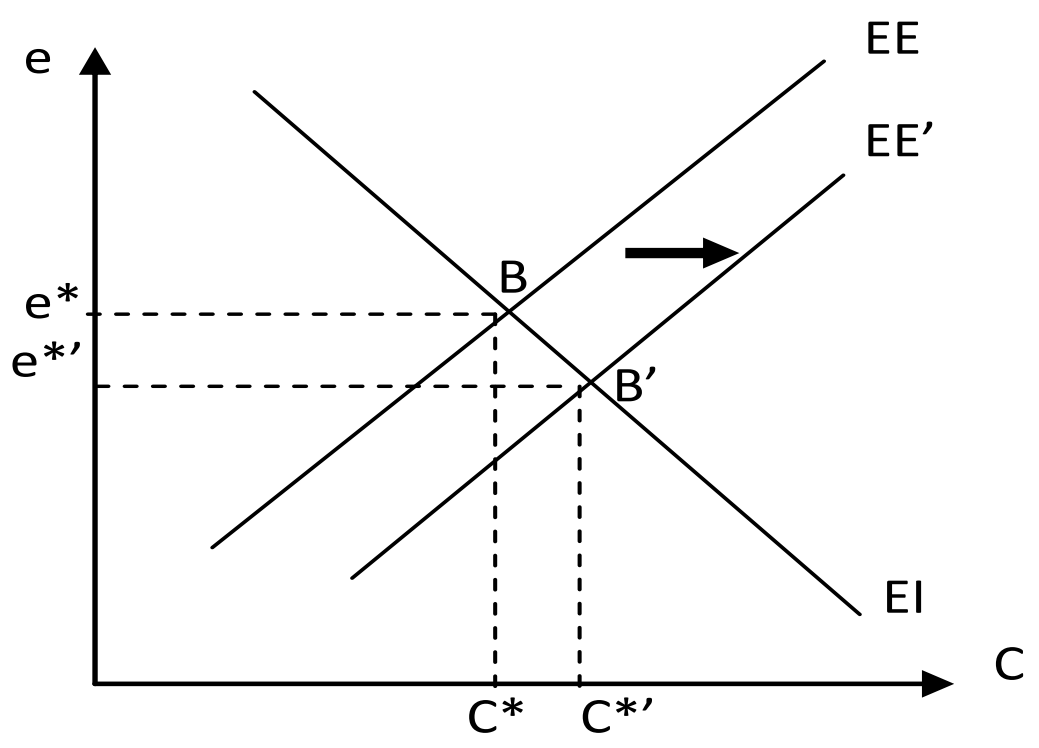

Par l'introduction de la variable transferts des fonds (trf), l'équation (3) d'équilibre externe devient :

$\dot{f}=b+t r f+Z+r f=Y_{E}(e, \rho)-G_{E}-(\theta+\tau) C+\operatorname{trf}+i d e+r f=0$

A partir des équations (2) et (4), nous déduisons le TCRE d'équilibre (e*) qui dépend de la balance commerciale, de la productivité, des coûts de transaction, des IDE, des transferts de fonds et de la consommation publique dans les deux types de biens.

$e=F\left(b, \rho, \tau, f, i d e, \operatorname{trf}, G_{n}, G_{E}\right)$

Or, pour faire intervenir les différentes variables dans le modèle, nous considérons le travail d'Ebadawi et Soto $(1994)^{5}$, où il a décomposé le prix des biens échangeable en prix des exportations et prix des importations, notés respectivement $\mathrm{P}_{\mathrm{x}}$ et $\mathrm{P}_{\mathrm{m}}$. Ce qui permet d'écrire : $P_{E}=Q\left[\left(1-t_{x}\right) P_{x}^{*}\right]^{\alpha} \cdot\left[\left(1+t_{m}\right) P_{m}^{*}\right]^{1-\alpha}$

\footnotetext{
${ }^{4}$ La courbe d'équilibre interne est notée EI.

${ }^{5}$ Le modèle d'Elbadawi et Soto (1994) est une extension des modèles de Rodriguez (1989) et d'Edwards (1987).
} 
$Q$ : Le taux de change nominal, $t_{x}, t_{m}$ sont des taxes des exportations et des importations respectivement. Ces taxes sont des mesures protectionnistes Bouoiyour et al. (2004). $P_{x}^{*}, P_{m}^{*}$ sont des prix internationaux à l'exportation et des à l'importation respectivement.

En remplaçant (6) dans (1) on trouve $e=\frac{P_{n}}{P_{E}}=\frac{P_{n}}{\mathrm{Q} P_{x}^{* \alpha} P_{m}^{* 1-\alpha}\left(1-t_{x}\right)^{\alpha}\left(1+t_{m}\right)^{1-\alpha}}$

Nous trouvons l'expression finale du TCR d'équilibre en fonction de ses fondamentaux.

$$
e=F\left(b, \rho, \tau, f, i d e, \operatorname{trf}, \frac{P_{x}}{P_{m}}, t_{x}, t_{m}, G_{n}, G_{E}\right)
$$

\section{Fondamentaux du taux de change réel effectif}

Nous retiendrons pour notre modèle les variables définies ci-après comme variables apparemment principales pour la Tunisie et liées à celles de l'équation (8): la productivité (pv), les termes d'échange (tot), la consommation publique (cpu), les transferts de capitaux sous forme des Investissements Directs Etrangers (ide), les transferts des fonds des travailleurs à l'étranger (trf), l'ouverture commerciale (ouv) et la position extérieure nette (nfa).

La productivité: les différences des taux de croissance de la productivité dans la production des biens échangeables d'un pays par rapport à celle des principaux partenaires commerciaux est un facteur potentiel qui affecte le TCRE. Ainsi, l'effet de Balassa-Samuelson est positif sur le taux de change réel. Il peut être interprété comme l'effet du développement économique sur le taux de change réel, c'est à dire les pays à croissance rapide ont tendance à connaître une appréciation réelle de leur taux de change Carrera et Restout (2004). Plusieurs études ont aussi montré un effet positif du progrès technique (Montiel, 1999; De Gregorio et Wolf, 1994; Hinkel et Montiel, 2001). Cependant le signe de cet effet dépend de l'effet qui domine: effet de l'offre ou la demande des biens non échangeables. Un effet positif se réalise que lorsque l'effet demande domine et vice versa (Edwards ,1989; Kalinda, 2001).

Les termes de l'échange: l'indice des termes de l'échange, est généralement défini comme le ratio de l'indice des prix à l'exportation par rapport à celui des prix à l'importation. Tous les 
modèles théoriques en général ${ }^{6}$ soulignent l'importance des perturbations des termes de l'échange comme une source potentielle des fluctuations des TCRE. La variation des termes d'échange entraîne un effet de substitution inter-temporelle et Intra-temporelle ainsi qu'un effet de revenu ${ }^{7}$ Eita et Sichei (2008). L'effet net sur le TCRE est ambigu et ne peut pas être connu à priori à cause de l'existence de ces deux effets Rano et Bayero (2008). Il dépend de l'effet qui domine: la dominance de l'effet revenu conduit à l'appréciation TCRE. Au contraire la détérioration des termes de l'échanges conduit à une dépréciation du TCRE (Zhang, 2001; Asfaha et Huda, 2002). Toutefois, des études empiriques récentes ont constaté que l'effet de revenu est prédominant (Elbadawi,1994 ; Baffes et al.,1999 ; Carrera et Restout,2004).

Les dépenses publiques: l'impact de la dépense publique sur le taux de change réel est ambigu et dépend de la composition sectorielle des dépenses Edwards (1989). En particulier, lorsque les dépenses du gouvernement s'effondrent de manière disproportionnée aux biens échangeables, l'augmentation des dépenses des secteurs des biens échangeables génère une augmentation de la demande d'importation qui se traduit par un déficit commercial qui à son tour nécessite une dépréciation réelle du taux de change pour maintenir l'équilibre de la balance extérieure. A contrario, l'augmentation des dépenses publiques principalement composées par des biens non échangeables exerce une pression à la hausse des prix relatifs des biens non échangeables et apprécie par la suite le taux de change réel. En conséquence, les pays ayant une grande part des dépenses publiques connaîtront aussi probablement une appréciation de leurs devises Froot et Rogoff (1991). Cependant cette variable peut être remplacée par la consommation totale lorsque le modèle s'attache beaucoup plus à la consommation sociale ou bien dans certains pays en développement qui n'ont pas des

\footnotetext{
${ }^{6}$ Par exemple Clark et MacDonald (1999), Elbadawi et Kahn (1997), Elbadawi (1994), Baffes et al. (1999), Carrera et Restout (2004) MacDonald et Ricci (2003), Egert, Halpern et MacDonald (2006), MacDonald et Dias (2007).

${ }^{7}$ L'effet revenu est lorsque le revenu intérieur augmente, l'amélioration des termes de l'échange va accroître la demande pour les biens non échangeables, en provoquant une nouvelle augmentation des prix afin de rétablir l'équilibre du marché Guillaumont et Hua (2002).

L'effet de substitution est lorsque les importations deviennent moins chères que les non-échangeables, la consommation des biens non-échangeables décroit et le prix des biens NE baisse pour absorber l'offre excédentaire des biens non échangeables, en conséquence le TCRE se déprécie.
} 
données sur la consommation publique en suivant les études de (Edwards, 1985, 1988 ; Chishti et Hasan, 1993).

L'ouverture commerciale: elle est introduite pour mesurer les restrictions commerciales. Selon Edwards, Elbadawi..., l'augmentation du degré d'ouverture implique une réduction des restrictions. Une libéralisation commerciale plus importante conduit à une dépréciation du TCR car elle permet l'augmentation des échanges et la convergence des prix (Goldfajn et Valdes, 1999 ; IEQ, 2003 ; Drine et Rault, 2005).

La position nette des actifs extérieurs est largement considérée dans la littérature comme une des variables déterminantes du TCRE d'un pays (MacDonald, 1997 ; MacDonald et Ricci, 2003). Lorsque la position nette extérieure d'un pays atteint un niveau plus élevé, une perte de compétitivité peut être maintenue, ce qui est associé à une appréciation du TCRE MacDonald et Ricci (2003). Un déficit du compte courant conduit à une augmentation de la dette extérieure nette qui peut être financée soit par les institutions financières internationales soit par les investissements étrangers à des taux de rendements élevés. Ceci peut conduire à une dépréciation de la monnaie Maeso-Fernandez et al. (2001).

Les Investissements Directs Etrangers sont considérés comme des flux de capitaux. Leur augmentation provoque une hausse de la demande des biens échangeables et non échangeables. Puisque les prix des biens échangeables sont des prix internationaux, les prix des biens non échangeables vont être augmentés suite à l'augmentation de la demande. Ce qui résulte une appréciation du TCRE (Krumm, 1993 ; Edwards, 1989, Edwards 1994).

Les transferts reçus de l'extérieure: l'impact macroéconomique de l'augmentation des transferts reçus de l'extérieure est une appréciation du TCRE par le biais de leurs effets positifs sur le solde du compte courant (Haque et Montiel, 1998 ; Izquierdo et Montiel, 2006). Ceteris paribus, les pays recevant les transferts des travailleurs connaissent une appréciation de leurs monnaies Amuedo-Dorantes C. et Pozo S. (2002). Selon Obstfeld et Rogoff (1996), ce genre de transfert de ressources nuit à la compétitivité du pays sur les marchés mondiaux.

\section{4) Analyse empirique}

Deux objectifs à entamer dans cette section. Le premier est d'estimer la valeur d'équilibre du TCRE du dinar tunisien moyennant le modèle d'Edwards précédemment discuté. Le second 
est de déduire l'impact des transferts de fonds des migrants tunisiens. Le vecteur des fondamentaux définis dans la section précédente, noté $v$ déterminant le TCRE.

$$
v=(p v, \text { tot }, \text { trf, ide, naf, ouv, } c p u)^{8}
$$

\section{Données}

Notre étude s'étale sur la période [1975, 2009]. Nous avons fait recours à plusieurs ressources afin de compléter notre base de données. Les statistiques descriptives des variables utilisées sont données par le tableau ${ }^{\circ} 1$. Aussi, leurs représentations graphiques sont données par la figure ${ }^{\circ} 4$ dans l'annexe. Le TCRE est disponible dans la base de données IFS (International Financial Statistics) de Fonds Monétaire International (FMI). Les dépenses publiques mesurées par rapport au PIB sont disponibles sur la base de données IFS. La productivité définie par le PIB par travailleur, est extraite de la base de données Maddison. Les termes de l'échange sont calculés par le ratio entre la valeur unitaire d'exportation et celle d'importation. Elles sont disponibles sur le site de la Banque Centrale de la Tunisie. L'ouverture commerciale définie par la somme des exportations et des importations divisée par le PIB, source IFS. Les transferts des fonds des travailleurs à l'étranger et les IDE sont mesurés par rapport au PIB, source Banque Mondiale. Enfin, La position nette des actifs extérieurs mesurée par rapport au PIB est définie par la somme cumulative de la balance courante depuis l'année 1967, source CHELEM-CEPII.

Tableau 1 : Statistiques descriptives

\begin{tabular}{|l|l|l|l|l|l|l|}
\hline Variables & Définition & Moyenne & Médiane & Écart-type & Minimum & Maximum \\
\hline le & Taux de change Réel Effectif & 4,862 & 4,766 & 0,238 & 4,561 & 5,363 \\
\hline lpv & Productivité & 9,706 & 9,677 & 0,172 & 9,444 & 10,054 \\
\hline ltot & Termes de l'Echange & 4,144 & 4,132 & 0,364 & 3,304 & 4,661 \\
\hline ltrf & Transferts de fonds & $-3,199$ & $-3,215$ & 0,164 & $-3,6$ & $-2,979$ \\
\hline lide & IDE & $-3,789$ & $-3,755$ & 0,653 & $-4,975$ & $-2,242$ \\
\hline nfa & La position nette des actifs extérieurs & $-1,083$ & $-1,17$ & 0,327 & $-1,537$ & $-0,29$ \\
\hline lcpu & Dépenses publiques & $-1,849$ & $-1,847$ & 0,052 & $-1,949$ & $-1,752$ \\
\hline louv & (Importations+Exportations)/PIB & $-0,373$ & $-0,37$ & 0,174 & $-0,664$ & 0,068 \\
\hline
\end{tabular}

\footnotetext{
${ }^{8}$ Les variables sont exprimées en logarithme népérien sauf la position extérieure nette. Ce qui mène à ajouter la lettre «l» au symbole de chaque variable transformée.
} 


\section{Stratégie d'estimation}

La procédure de modélisation économétrique adoptée dans notre travail repose sur deux propriétés théoriques importantes relatives à l'équilibre de long terme. La première est l'existence à long terme d'une relation à l'état stationnaire entre le taux de change réel effectif et ses déterminants fondamentaux réels. Ce qui revient à poser une forme linéaire ${ }^{9}$ de la manière suivante: $e_{t}=\beta_{0}+\beta^{\prime} v_{t}+\varepsilon_{t} ; \varepsilon_{t}$ sont des résidus Identiquement et Indépendamment Distribué (IDD). Dans la mesure de prouver l'existence des relations d'équilibre de long terme, la méthode de cointegration sera adoptée. Les tests de cointégration les plus utilisées dans la détermination de la présence d'une telle relation sont ceux d'Engle et Granger (1987) et de Johansen $(1988,1991)$ dans le cas sans rupture. Or nous allons tenir compte des ruptures dans notre étude, donc nous adopterons la méthode de Johansen, Mosconi et Nielsen (2000, noté JM\&N), Saikkonen et Lütkepohl (2000, noté (S\&L)) et Saikkonen, Lütkepohl et Trenkler (2004, noté (SL\&T)). La deuxième est d'avoir un état stationnaire dynamiquement stable $^{10}$. Pour répondre à une telle situation, nous adoptons le modèle à correction d'erreur suivant:

$$
\Delta e_{t}=\alpha\left(e_{t-1}-\beta_{0}-\beta^{\prime} v_{t-1}\right)+\sum_{j=1}^{p} K_{t} \Delta e_{t-j}+\sum_{j=0}^{p} \lambda_{j}^{\prime} \Delta v_{t-j}+\mu_{t}
$$

$\Delta:$ opérateur de différence, $\mu_{t}:$ résidus (IDD), $\alpha$ : terme de correction d'erreur, $j:$ retard. $^{11}$

Nous notons ici que $\alpha$ est la vitesse d'ajustement automatique vers l'état stationnaire. D'après Baffes et al. (1999), l'équilibre de long terme est stable lorsque la condition $-2<\alpha<0$ est vérifiée. Avant de passer à l'estimation proprement dite, le test de stationnarité est indispensable.

\section{Test de racine unitaire avec ruptures}

Etant donné la forte relation qui existe entre le test de stationnarité et la cointégration, nous trouvons dans la littérature actuelle un bon nombre de travaux qui traitent de la cointégration en présence du break dans la tendance. A titre d'exemple nous citons Gregory et Hansen

\footnotetext{
${ }^{9}$ Cette relation est valable que lorsque les variables ont le même ordre d'intégration (Haorau (2006)). C'est-àdire sont tous I $(1)$.

${ }^{10}$ Suite à un choc exogène incitant le taux de change réel effectif à s'écarter de son niveau d'équilibre stationnaire à court terme, les mécanismes auto-correcteurs doivent se mettre en place pour produire une convergence du système vers l'équilibre stationnaire (Haorau (2006)).

${ }^{11}$ Le retard optimal est choisi selon les critères suivant : Akaike Info Criterion, Final Prediction Error, HannanQuinn Criterion, Schwarz Criterion.
} 
(1996), Inoue (1999), Lee et Strazicish (2001), JM\&N(2000), S\&L (2000) et SL\&T (2004). En tenant compte de cette innovation dans les études des séries temporelles, la mise en œuvre des tests de stationnarité avec rupture de notre travail sera faite sur la base de la méthode de S\&L (2002) et Lanne et al. (2002) qui a pour avantage de ne pas supposer connue a priori la date de rupture. Ensuite l'hypothèse de coïntégration sera testée, comme nous avons cité cidessus, à l'aide des méthodes de JM\&N (2000), S\&L (2000) et SL\&T (2004) qui admettent la présence de ruptures dans la relation de coïntégration. Le logiciel JMulTi sera l'outil de tous ces tests. Les résultats des tests de racine unitaire ave coupures des variables du modèle sont présentés dans le tableau $n^{\circ} 2$ suivant :

Tableau 2: Test de racine unitaire avec Break

\begin{tabular}{|l|c|l|l|l|ll|}
\hline Variables & Breaks & Shift dummy & Exponentiel Shift & Rational Shift & \multicolumn{2}{|l|}{ Conclusion } \\
\hline le & 1986 (a) & -1.862 & -2.001 & 0.207 & I(1) & \\
& (b) & -1.531 & -1.566 & -1.712 & & \\
\hline Ipv & 1984 (a) & 0.979 & 0.994 & 1.885 & I(1) & \\
& 1986 (b) & -1.763 & -1.834 & -2.716 & & \\
\hline Itot & 1996 (a) & -1.822 & -1.786 & -1.611 & I(1) & \\
& (b) & -1.969 & -1.933 & -2.224 & & \\
\hline lcpu & 1990 (a) & -1.558 & -1.503 & -1.794 & I(1) & \\
& (b) & -1.170 & -1.087 & -1.739 & & \\
\hline lide & 1992 (a) & -2.522 & -2.477 & $-3.128 * *$ & I(1) & ou \\
& (b) & -2.508 & -2.488 & $-3.047 * *$ & I(0) & \\
\hline Irf & 1994 (a) & -1.898 & -1.808 & $-3.595 * * *$ & I(1) & ou \\
& (b) & -1.489 & -1.435 & -1.936 & I(0) & \\
\hline Nfa & 1993 (a) & -2.608 & -2.657 & $-4.502 * * *$ & I(1) & ou \\
& (b) & -1.327 & -1.395 & -2.084 & I(0) & \\
\hline louv & 1989 (a) & -2.652 & -2.612 & -1.917 & I(1) & ou \\
& 1985 (b) & $-3.847 * * *$ & $-3.879 * * *$ & $-2.791 *$ & I(0) & \\
\hline
\end{tabular}

1- (a) Sans tendance. (b) Avec tendance.

2- Les valeurs critiques tirées de Lanne et al. (2002) pour les seuils $1 \%$, $5 \%$, et $10 \%$ sont respectivement,-3,48, -2,88, et -2,58 pour le modèle sans tendance, et -3,55, -3,03, et -2,76 pour le modèle avec tendance.

3-Les astériks ***, ** et * indiquent respectivement la stationnarité aux seuils $1 \%$, $5 \%$, et $10 \%$.

D'après ce tableau ci-dessus, nous constatons que l'l'hypothèse de non-stationnaire domine dans la plupart des cas. Dans certains cas, la conclusion n'est pas claire : nous pouvons retenir soit I(1) soit I(0). Dans ce cas, nous suivons le travail de Jaussaud et Rey (2009) et nous considérons ces variables comme non stationnaires. 


\section{Test de cointégration}

Les résultats des tests de stationnarité déjà trouvés nous permettent en conséquence de passer à l'étape suivante qui teste la présence de relation de cointégration entre le TCRE et ses fondamentaux. Le test de cointégration a été fait à l'aide des tests développés par JM\&N et S\&L. Ainsi, des variables muettes (dummy) qui reflètent les dates de breaks mises en évidence par les tests précédents, ont été introduites. L'analyse des résultats du test de cointégration du modèle montre que le ratio de vraisemblance indique que l'hypothèse nulle de non cointégration est rejetée en faveur de l'hypothèse alternative d'existence d'un vecteur de cointégration. Ce qui indique l'existence d'une relation de cointégration. Le tableau $\mathrm{n}^{\circ} 3$ donne les résultats du test de cointégration de $S \& L$ avec la présence d'un trend et des breaks.

\begin{tabular}{|l|l|l|l|l|l|}
\hline \multicolumn{5}{|c|}{ Tableau 3: Résultats des tests de cointégration selon S\&L du modèle } \\
\hline r0 & LR & Pval & $\mathbf{9 0 \%}$ & $\mathbf{9 5 \%}$ & $\mathbf{9 9 \%}$ \\
\hline $\mathbf{0}$ & $\mathbf{1 5 5 , 5 5}$ & $\mathbf{0 , 0 3 3 3}$ & $\mathbf{1 4 7 , 0 4}$ & $\mathbf{1 5 2 , 5 9}$ & $\mathbf{1 6 3 , 3 8}$ \\
\hline 1 & 111,36 & 0,1541 & 114,84 & 119,77 & 129,39 \\
\hline 2 & 80,82 & 0,2202 & 86,64 & 90,95 & 99,4 \\
\hline 3 & 55,45 & 0,291 & 62,45 & 66,13 & 73,42 \\
\hline 4 & 30,09 & 0,6414 & 42,25 & 45,32 & 51,45 \\
\hline 5 & 20,36 & 0,3679 & 26,07 & 28,52 & 33,5 \\
\hline 6 & 6,84 & 0,681 & 13,88 & 15,76 & 19,71 \\
\hline 7 & 0,26 & 0,9712 & 5,47 & 6,79 & 9,73 \\
\hline Note : & Les breaks s84 s90 s92 s94 correspondant respectivement aux changements [1984] \\
[1990] [1992] [1994] ont été introduits.
\end{tabular}

\section{Relation de long terme}

La détermination de la relation de cointégration trouvée a été faite par l'intermédiaire d'une estimation du modèle des vecteurs à correction d'erreur où il n'y a ni variables exogènes ni restrictions sur la dynamique de court terme. Toutefois, nous pouvons attribuer à la relation de cointégration différents termes déterministes; une constante, un trend et/ou des variables muettes. L'estimation de la relation de cointégration est donnée par l'équation ci-dessous ${ }^{12}$.

$$
\begin{aligned}
& l e=\underbrace{0,96 l p v}_{(6,65)^{* * * * * *}}+\underbrace{0,02 l \text { lot }}_{(0,26)}+\underbrace{0,39 l \text { trf }}_{(7,77)^{* * * * *}}+\underbrace{0,07 l i d e}_{(9)^{* * * * *}}-\underbrace{0,19 n f a}_{(-3,6)^{* * * * * *}}-\underbrace{1,17 l \text { louv }}_{(-25,8)^{* * * * *}}-\underbrace{2,33 l c p u}_{(-13,24)^{* * * * *}}-\underbrace{0,09 s 84}_{(-4,70)^{* * * * *}} \\
& +\underbrace{0,25 s 90}_{(11,95)^{* * * * *}}+\underbrace{0,08 s 94}_{(5,21)^{* * * * *}}-\underbrace{7,23}_{(-5,16)^{* * * * *}}-\underbrace{0,04 \text { Trend }}_{(-12,78)^{* * * * *}}
\end{aligned}
$$

12 - Entre parenthèse t student, *** significativité à 1\%, ** significativité à 5\%. 
Nous constatons qu'à l'exception des termes d'échange, tous les coefficients des variables sont statistiquement significatifs et différents de 0 , indiquant en conséquence un impact significatif sur le TCRE. Etant donné que toutes les variables sont exprimées en forme logarithmique à l'exception de la variable position extérieure nette, tous ces coefficients sont alors des élasticités. Donc, une augmentation de $1 \%$ de transferts provoque une appréciation de taux de change réel d'équilibre de $0,39 \%$. Ce résultat confirme l'hypothèse de syndrome hollandais dans l'économie tunisienne. Nous complétons l'analyse du modèle estimé par une étude de la fonction réponse du TCRE à un choc des transferts des fonds des travailleurs. La figure $\mathrm{n}^{\circ} 7$ dans l'annexe, donne la réponse de TCRE d'équilibre suite à un choc sur les envois des fonds des travailleurs. La figure $n^{\circ} 8$, donne les réponses cumulées. Nous constatons une appréciation réelle du TCRE à long terme, ce qui est cohérent avec le modèle théorique. Cependant, la réponse de court terme est négative cela peut s'expliquer par la présence des rigidités à l'hausse des biens non échangeables à court terme et les interventions des autorités monétaires sur le marché de change pour stabiliser le taux de change. Ce type de réponse à court terme peut nous pousser à penser que les tunisiens thésaurisent les transferts de fonds à court terme dans le but des investissements futures. Ce dernier comportement engendre une balance commerciale excédentaire et une accumulation des réserves de change. Dans ce cas le processus d'ajustement réel de l'économie se réalise à long terme.

\section{Mésalignement}

Avant de calculer le mésalignement, nous allons déduire la valeur d'équilibre de taux de change réel sur la base de taux change réel estimé de dinar tunisien. La valeur d'équilibre de taux de change réel d'Edwards est obtenue en remplaçant les valeurs d'équilibre des variables fondamentales dans l'équation estimée. Ces valeurs ont été obtenues en utilisant la moyenne mobile. La courbe de taux de change réel d'équilibre calculé est donnée par la figure $\mathrm{n}^{\circ} 5$ dans l'annexe. Le mésalignement de taux de change réel qui n'est autre que la différence entre le TCRE d'équilibre et le TCRE observé. Lorsque la valeur de mésalignement est positive, le dinar tunisien est sous-évalué. Dans le cas contraire, le taux de change est sur-évalué. D’après la figure $\mathrm{n}^{\circ} 6$ de mésalignement, nous constatons qu'avant l'adoption de Plan d'Ajustement Structurel (PAS) en 1986, la déviation du TCRE par rapport à sa valeur d'équilibre est très importante. Cet écart ne peut que renforcer l'instabilité de taux de change réel et aggraver la crise économique de pays qui s'est terminé par une dévaluation du dinar tunisien dans le cadre du PAS. Avant l'année de 1986, nous constatons en général une surévaluation du dinar 
tunisien, sauf pour quelques années (allant de 1978 jusqu'au 1980), qui atteint sa valeur maximale en 1985, soit un taux de 30.4\%. Après le PAS, le dinar tunisien a connu des comportements inverses en enregistrant en grosso modo une sous-évaluation dont le taux moyen de Mésalignement de la période [1986-2009] est de -2.8\%. En s'appuyant sur le figure du Mésalignement mous pouvons clairement constater qu'après le PAS, le Taux de change réel est proche à sa valeur d'équilibre. Ce qui est conforme avec les conclusions du FMI (2010) qui affirme que le TCRE est relativement stable et est globalement en ligne avec les fondamentaux.

\section{4) Conclusion}

Ce travail s'inscrit dans le cadre d'estimation de taux de change réel d'équilibre de la Tunisie à long terme. L'impact des variables explicatives choisies par notre modèle du TCRE est consistant. Une augmentation des variables suivantes : productivité, transferts de fons des travailleurs tunisien à l'étranger, IDE conduira à une appréciation de change réel d'équilibre. En ce qui concerne la position extérieure nette, dépenses publiques et ouverture commerciale leur augmentation engendrera une dépréciation de taux de change réel. L'idée centrale dans ce travail est vérifier l'existence de syndrome hollandais engendré par les transferts de fonds. L'estimation de l'équation de long terme montre cet effet. En d'autres termes, les transferts de fonds des travailleurs tunisiens à l'étranger provoquent une appréciation de taux de change réel d'équilibre. Ce qui approuve l'hypothèse de syndrome hollandais. Ceci peut affaiblir la compétitivité extérieure. A cet égard, les transferts de fonds imposent aussi un coût économique non-intentionnel sur le pays bénéficiaire Amuedo-Dorantes et Pozo (2004). Il y a lieu de signaler que plusieurs études suggèrent que les transferts de fonds ont deux types d'effets opposés: des effets bénéfiques et des autres préjudiciables Funkhouser (1992). D’un coté, ces fonds ont tendance à faire baisser le taux global d'activité en réduisant la nécessité pour certains membres de la famille de participer au marché du travail. D’autre part, ils permettent également de promouvoir l'auto-emploi et contribuent à alléger les contraintes financières résultant des imperfections des marchés du crédit. De même, ils participent à la réduction du risque de décrochage scolaire pour les enfants de familles rurales Cox Edwards et Ureta (2003). Enfin, concernant le mésalignement nous avons trouvé l'écart entre le taux de change observé et celui d'équilibre est très faible notamment depuis la mise en place du PAS. Ce qui signifie que les autorités monétaires, appliquent une politique du taux de change très prudente. 


\section{Bibliographie}

Amuedo-Dorantes, C., Pozo, S., (2004). "Workers' remittances and the real exchange rate: a paradox of gifts". World development. Vol. 32 (8). Pg 1407-17.

Amuedo-Dorantes, C., Pozo. S., (2002)."Precautionary Saving by Young Immigrants and Young Natives".Southern Economic Journal 69, pp. 48-71.

and the Dutch Disease: A Panel Data Analysis". Federal Reserve Bank of Atlanta Working Paper 2008-12.

Asfaha, S.G., Huda, S.N., (2002). "Exchange Rate Misalignment and International Trade Competitiveness: A Cointegration Analysis for South Africa". Paper Presented at the TIPS Annual Forum at Glenburn Lodge, Muldersdrift.

Baffes J., A.I Elbadawi., S.A. O'Connell (1999). Single-equation of the equilibrium real exchange rate, dans HINKLE L.E. et MONTIEL P.J. éds., "Exchange rate misalignment: concepts and measurement for developing countries" World Bank Research Publication, 405-464.

Banque Mondiale (2011) "Migration and Remittances Factbook 2011-Tunisia" http://siteresources.worldbank.org/INTPROSPECTS/Resources/3349341199807908806 /Tunsia.pdf

Barajas, A., Chami, R., Hakura, D., Montiel, P., (2010). "Workers' Remittances and the Equilibrium Real Exchange Rate: Theory and Evidence”. IMF Working Papers 10/287, International Monetary Fund.

Bouoiyour J., Marimoutou V. et Rey S. (2004). Taux De Change Réel D’équilibre Et Politique De Change Au Maroc: Une Approche Non Paramétrique. Économie internationale 97, p. 81-104.

Bourdet, Y., Falck, H., (2006). "Emigrants' Remittances and Dutch Disease in Cape Verde". International Economic Journal, 20: 267-284.

Carrera J., Restout, R., (2004). "Long run determinants of real exchange rates in Latin America". W.P. 08-11. Groupe d'Analyse et de Théorie Économique (GATE). UMR 5824 du CNRS. gate@gate.cnrs.fr.

Chishti., S., Hasan, M. A. (1993). "What Determines the Behavior of Real Exchange Rate in Pakistan". The Pakistan Development Review, 32(4).

Clark, P., MacDonald, R., (1998). "Exchange Rate and Economic Fundamentals: A Methodology Comparison of BEERs and FEERs," IMF Working Paper 98/67 (Washington: International Monetary Fund).

Clark, P.B., MacDonald, R., (1999). "Exchange Rates and Economic Fundamentals: A Methodological Comparison of BEERs and FEERs. In R. MacDonald and J Stein (eds) 
Equilibrium Exchange Rates, Kluwer: Amsterdam. And IMF Working Paper 98/67 (Washington: International Monetary Fund, March 1998).

Corden, W. M., Neary, J.P., (1982). "Booming Sector and Deindustrialization in a Small Open Economy.” Economic Journal, Vol. 92, n³68, p. 825-48.

Corden, W.M., (1984). "Booming Sector and Dutch Disease Economics: Survey and Consolidation." Oxford Economic Papers, Vol. 36, p. 359-380.

Cox Edwards, A., Ureta, M., (1999). "International migration, remittances, and schooling: evidence from El Salvador”. Journal of Development Economics 72 (2003) 429- 461.

De Gregorio, J., Wolf, H., (1994). "Terms of Trade, Productivity, and the Real Exchange Rate", NBER Working Papers 4807.

Drine I., Rault. C., (2005). "Déterminants de long terme des taux de change réels pour les pays en développement : une comparaison internationale". Revue d'économie du développement. Vol. 19. Pg 123-150.

Edwards S. (1987). Tariffs, Terns of Trade and Real Exchange Rate in an Intertemporal Model of the Current Account," NBER Working Paper.

Edwards, S., (1985). "The behavior of interest rates and the real exchange rates during a liberalization episode: the case of Chile 1973-83". NBER Working Paper No 1702. September.

Edwards, S., (1989). "Real exchange rates. Devaluation and adjustment: exchange rate policy in Developing Countries”. MIT Press. Cambridge. Massachusetts.

Edwards, S., (1994). "Real and monetary determinants of real exchange rate behavior: theory and evidence from developing countries". In estimating equilibrium exchanges rates. Williamson, J., eds., Institute for International Economics, Washington.

Edwards. S., (1988). “Exchange Rate Misalignment in Developing Countries". Published for The World Bank, Occusional paper $n^{\circ} 2 /$ new series, the Johns Hopkins Unibersity Press, Baltimore MD.

Egert, B., Halpern, L., MacDonald, R., (2006). "Equilibrium Exchange Rates in Transition Economies: Taking Stock of the Issues". Journal of Economic Surveys 20(2), 257-324.

Eita, J., Sichei, M., (2006). "Estimating the Equilibrium Real exchange rate for Namimbia". Department of Economics working paper series 2006-08, University of Pretoria.

Elbadawi, I. A., Soto, R., (1994). "Capital flows and long-term equilibrium real exchange rates". In chile. Policy research working paper $n^{\circ} 1306$. The World Bank. Macroeconomics and growth division. June. 
Elbadawi, I.A., Kahn, B., (1997). "Determinants of the Real Exchange Rate in South Africa". Working Paper, WPS/97-16 (Oxford: Oxford University, Centre for the Study of African Economies).

Engle, R., Granger, C., (1987). "Co-Integration and Error-Correction: Representation, Estimation, and Testing“. Econometrica, vol. 35, pp. 251-276.

Fajnzylber, P., López, H., (2007). "Close to Home: The Development Impact of Remittances to Latin America”. World Bank, Washington DC.

Froot K.A. \& K. Rogoff (1991), « The EMS, the EMU, and the Transition to a Common Currency », NBER Macroeconomic Annual 1991, pp. 269-317.

Funkhouser E. (1992), "Migration from Nicaragua: Some Recent Evidence," World Development 20 (August): 1209-18.

Goldfajn, I., Valdés R., (1999). "The aftermath of appreciations", Quarterly Journal of Economics 114: 229-62.

Grabel, I., (2008). "The Political Economy of Remittances: What Do We Know? What Do We Need to Know?" Political Economy Research Institute Working Paper series $\mathrm{N}^{\circ}$ 184.

Gregory, A. and B. Hansen, 1996. "Residual-Based Tests for Cointegration in Models with Regime Shifts", Journal of Econometrics, 70, 99-126.

Guillaumont J. S., Hua. P., (2002). "The Balassa-Samuelson effect and inflation in the Chinese provinces”. China Economic Review, 13, 2-3, 134-160.

Haque, N. U., Montiel, P. J., (1998), "Exchange Rate Policy in Pakistan: Recent Experience and Prospects". In Nasim Anjum (eds), Financing Pakistan's Development in the 1990s, Oxford University Press.

Hinkel, L.E., Montiel, P.J., (2001). "Exchange Rate Misalignment, Concepts and Measurement for Developing Countries'. Oxford University Press.

Hoarau, J. F., (2006). "Les régimes monétaires en Australie, 1977-2000 : une mise en perspective à l'aide d'un indicateur de distorsion du taux de change réel". Économie internationale. 105. Pg. 85-112.

IEQ., (2003). "Évolution du taux de change du Dinar (1961-2000) (Une approche par le taux de change d'équilibre) ". République Tunisienne, Ministère du Développement et de la Coopération Internationale. Les cahiers de 1'INSTITUT D'ECONOMIE QUANTITATIVE (IEQ). Nº17. Mars. Pg. 69-116.

Inoue, A., (1999). Tests of Cointegrating Rank with a Trend-Break. Journal of Econometrics 90, 215-237. 
Izquierdo, A., Montiel, P. J., (2006). "Remittances and equilibrium real exchange rates in six Central American Countries”. Williams College mimeo.

Jaussaud, J., Rey S.,(2009), "Long-Run Determinants of Japanese Import Flows from USA and China : A Sectoral Approach”. Document de travail CATT n ${ }^{\circ}$, novembre.

Johansen, S., (1988). "Statistical analysis of co-integration vectors". Journal of economic dynamics and control. Vol: 12. Pg 231-254.

Johansen, S., (1991). "Estimation and hypothesis testing of cointegration vectors in gaussian vector autoregressive models". Econometrica. Vol. 52. Pg 389-402.

Johansen, S., Mosconi, R., Nielsen, B., (2000). "Cointegration analysis in the presence of structural breaks in the deterministic trend". Econometrics Journal 3: 216-249.

Kalinda, M. B. (2001). "Long-run and Short-run Determinants of the Real Exchange Rate in Zambia", Scandinavian Working Papers in Economics, ${ }^{\circ}{ }^{\circ}$, Université de Göteborg, Suède

Kapur, D., (2004). "Remittances: The New Development Mantra?" G-24 Discussion Paper No. 29.

Krugman, P. R., Obstfeld, M. (2007). "Economie Internationale" Edition Person Education France. ISBN : 2-7440-7140-4

Krumm, K. L., (1993), “A Medium-Term Framework for Analyzing the Real Exchange Rate, with Applications to the Philippines and Tanzania", The World Bank Economic Review, Vol. 7, n 2, Pg.219-245.

Lanne, M.., Lütkepohl, H., Saikkonen. P., (2002). Comparision of unit root tests for time series with level shifts. Journal of Time Series Analysis. 23. Pg: 667-685.

Lartey, E., Mandelman, F. and Acosta P., (2008). "Remittances, Exchange Rate Regimes, and the Dutch Disease: A Panel Data Analysis". Federal Reserve Bank of Atlanta Working Paper 2008-12. Atlanta.

Lee, J., Strazicich, MC., (2001). "Break point estimation and spurious rejections with endogenous unit root tests". Oxford Bulletin of Economics and Statistics, 63(S1), pp.535-558.

MacDonald, R., (1997). "What determines real exchange rates ? The long and short of it". IMF Staff Papers, 97/21, Washington, D.C. IMF, April.

MacDonald, R., Dias, P., (2007). "Behavioural equilibrium exchange rate estimates and implied exchange rate adjustments for ten countries". University of Glasgow Working Paper No. 12. 
MacDonald, R.,Ricci, L., (2003). "Estimation of the equilibrium real exchange rate for South Africa”. IMF Working Paper, 2003/ 44, Washington, D.C., IMF.

Maeso-Fernandez, F., Osbat, C., Schnatz. B., (2002). "Determinants of the euro real effective exchange rate: A BEER/PEER approach". Australian Economic Papers 41(4), 437-461.

Makhlouf, F., Mughal, M., (2010). "Remittances and Dutch disease in Pakistan: A Bayesian approach”. International Economics Conference, June 2010, Hammamet Tunisie.

Mongardini, J., Rayner.B., (2009). "Grants, Remittances, and the Equilibrium Real Exchange Rate in Sub-Saharan African Countries”. IMF Working Paper No. 09/75.

Montiel, P. J., (1999). "Determinants of the long-run equilibrium real exchange rate: an analytical model”. In Hinkle, L., Montiel, P. (1999). Exchange rate misalignment: concepts and measurement for Developing Countries. (Oxford: Oxford University Press).

Mughal, M., Makhlouf, F., (2011). "Volatility of Remittances to Pakistan: What do the Data Tell?” Economics Bulletin, AccessEcon, vol. 31(1), pages 605-612.

Obstfeld, M., Rogoff.K., (1996). "Foundations of International Macroeconomics". Cambridge, MA: MIT Press.

Rajan, R.G., Subramanian, A., (2010). “Aid, Dutch disease, and manufacturing growth”. Journal of Development Economics.

Rano, S. U., Bayero. A., (2008). "Real Exchange Rate Misalignment: An Application of Behavioral Equilibrium Exchange Rate to Nigeria”. MPRA Paper $n^{\circ} 10343$. September.

Rapport du FMI (2010). N 10/282 en septembre.

Ratha. D., (2003). "Workers' Remittances: An Important and Stable Source of External Development Finance" in Global Development Finance, Ch. 7, World Bank.

Rodriguez, C. A., (1989). "Macroeconomic Policies for Structural Adjustnent". Working Papers Series ${ }^{\circ} 247$. The World Bank.

Saikkonen, P., Lütkepohl, H., (2000). "Testing for the cointegrating rank of a VAR process with structural shifts”. Journal of Business \& Economic Statistics. 18. Pg 451-464.

Saikkonen, P., Lütkepohl, H., (2002). "Testing for a unit root in a time series with a level shift at unknown time". Econometric Theory. 18. Pg 313-348.

Saikkonen, P., Lütkepohl, H.,Trenkler,C., (2004). "Break Date Estimation and Cointegration Testing in VAR Processes with Level Shift". Site internet: http://www.eui.eu/Personal/Luetkepohl/mshift-4_1.pdf. 
Singer, D.A., (2010). "Migrant Remittances and Exchange Rate Regimes in the Developing World”. American Political Science Review, Vol. 104, No. 2, Forthcoming.

Vargas-Silva, C., (2007). "The Tale of Three Amigos,Remittances, Exchange Rate and Money Demand in Mexico”. SHSU Economics \& Intl. Business Working Paper No. SHSU_ECO_WP07-04 September 2007.

Williamson, John,1983, The Exchange Rate System, Policy Analyses in International Economics, No. 5, Washington: Institute for International Economics, September .. (Also Revised Edition, June 1985.)

Zhang, Z., (2001). "Real Exchange Rate Misalignment in China: An Empirical Investigation". Journal of Comparative Economics, No. 29, pp. 80-94. 


\section{Annexes}

Figure 1 Transferts de fonds en Tunisie en millions de dollars courants (1976-2009)

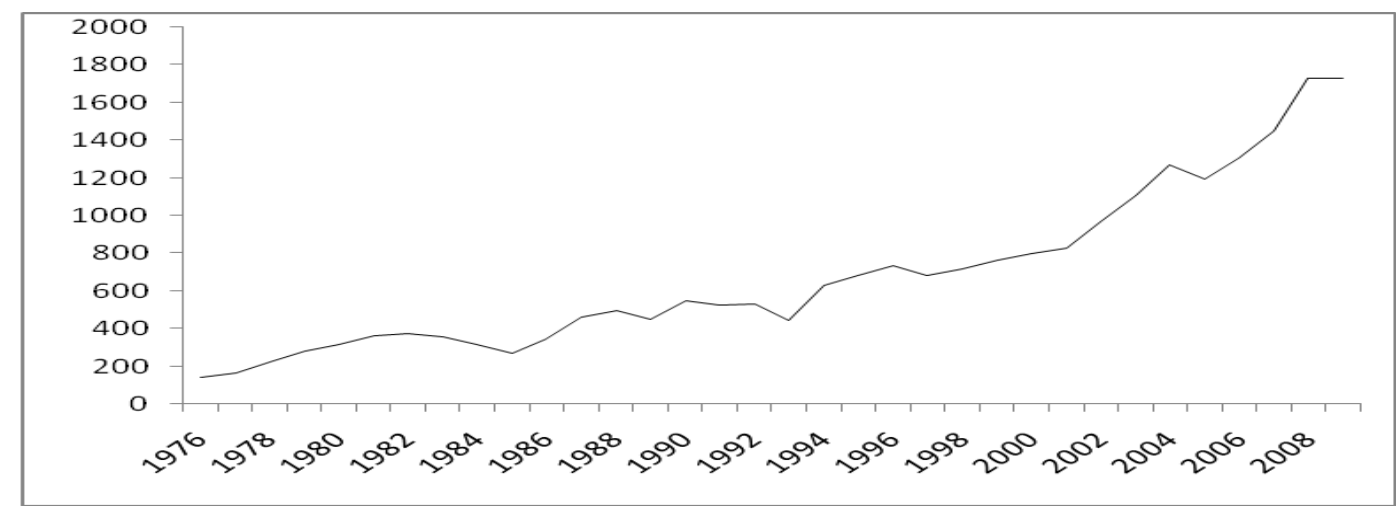

Sources: World Bank, World Development Indicator (WDI, 2011)

Figure2 : Evolution des flux financiers vers la Tunisie (1976-2009)

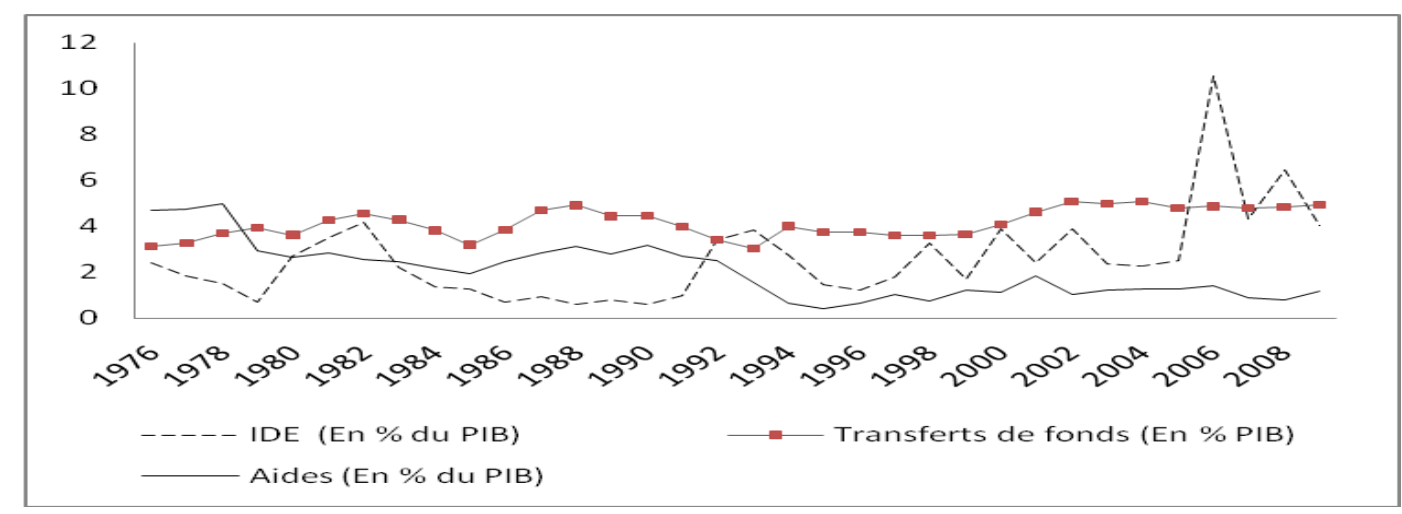

Sources: World Bank, World Development Indicator (WDI, 2011)

Figure3 Consommation des ménages en fonction des transferts de fonds (1976-2009)

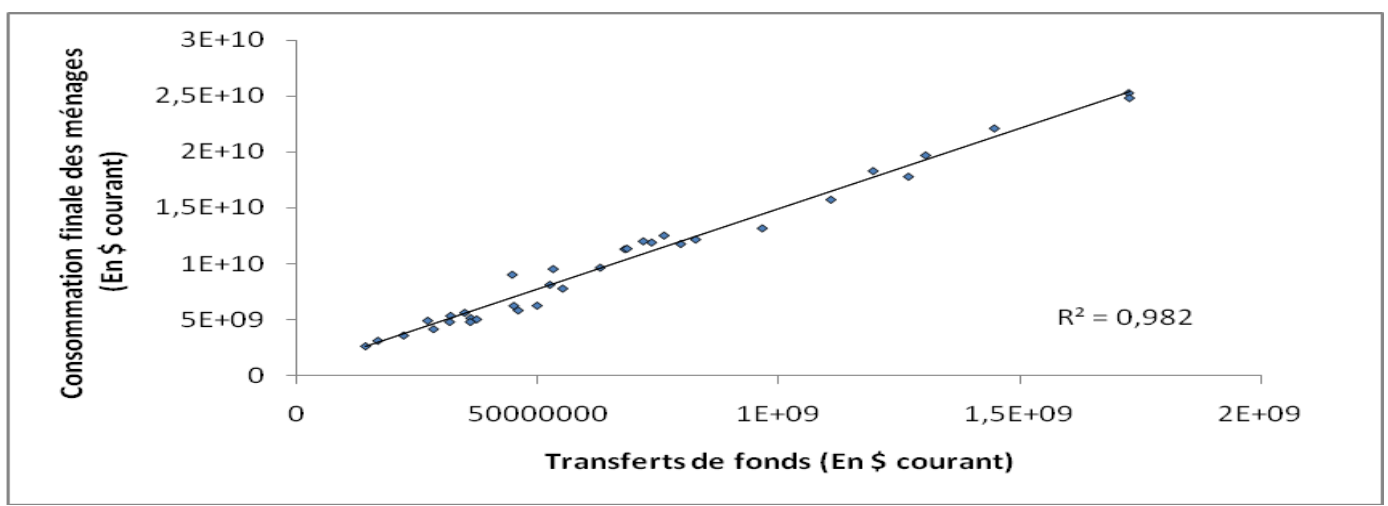

Sources: World Bank, World Development Indicator (WDI, 2011) 
Figure 4 : Taux de change et ses fondamentaux.
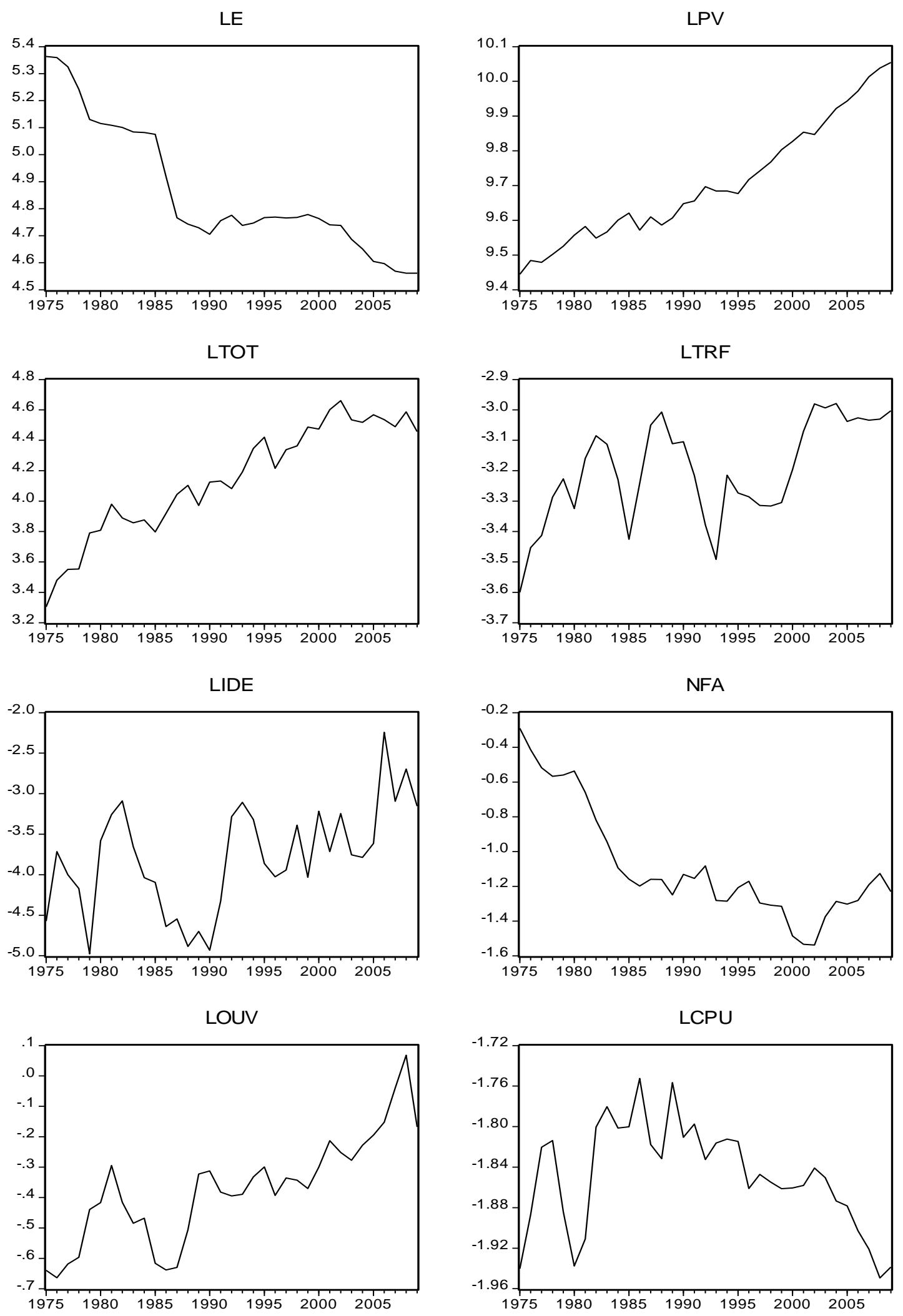
Figure 5 : Taux de change observé et taux de change d'équilibre

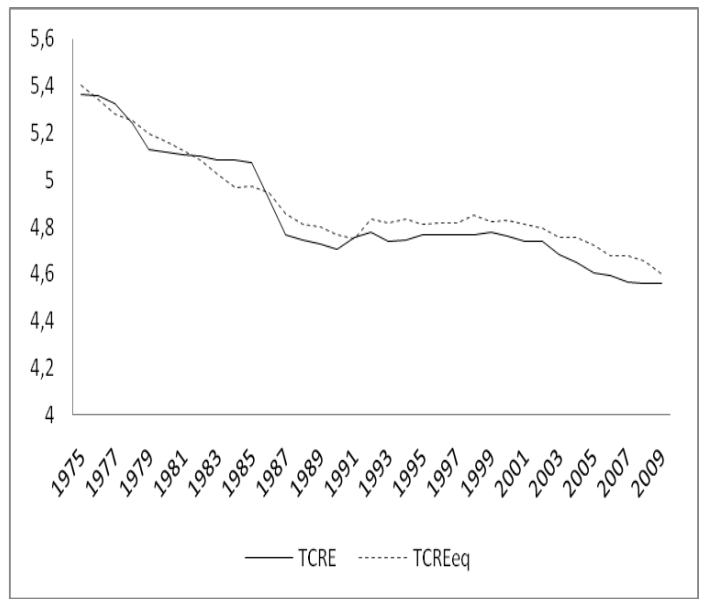

Figure 7 :Réponses de taux de change suite à un choc des transferts

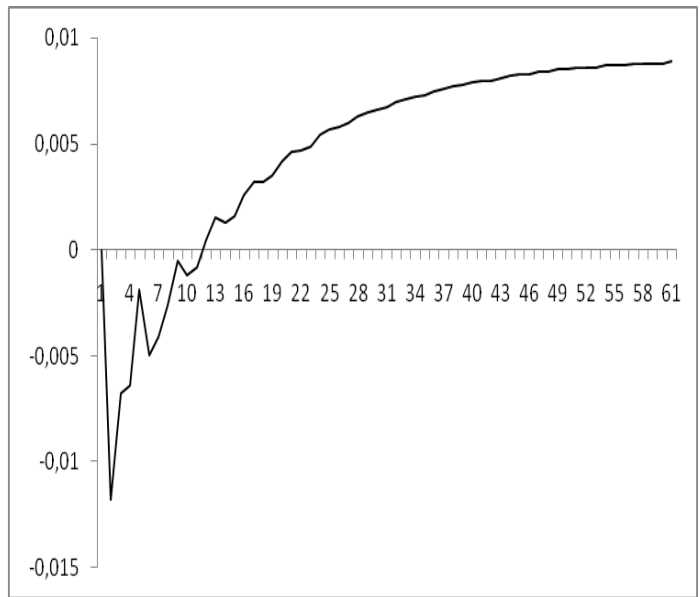

Figure 6 : Mésalignement

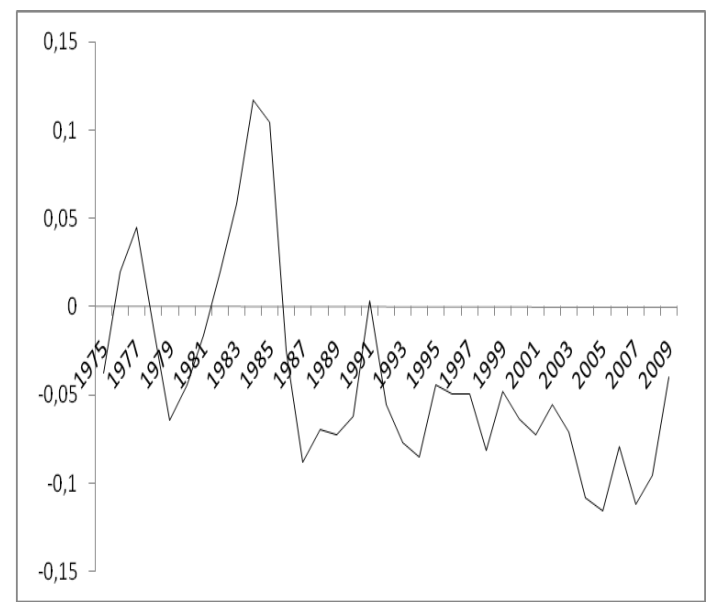

Figure 8 : Réponses cumulées

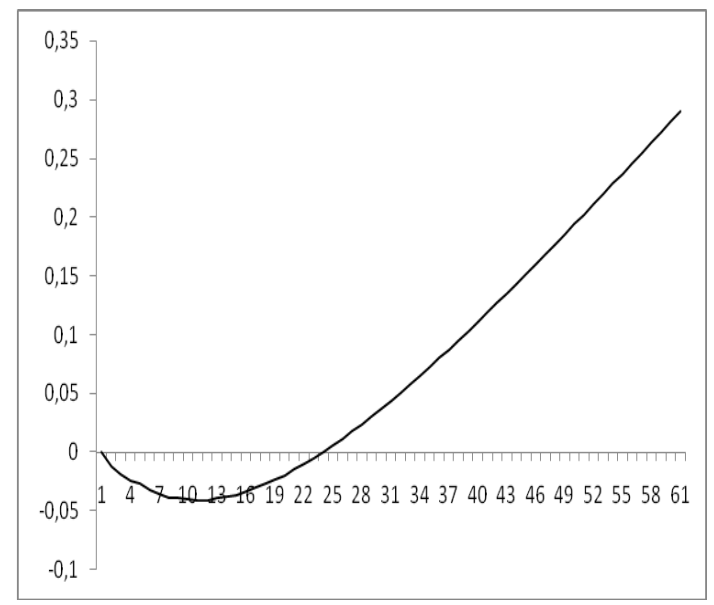

